\title{
Comparison of urokinase and reteplase thrombolytic treatment in patients with high-risk pulmonary embolism
}

\author{
YI ZHANG ${ }^{1}$, LAN MA $^{2}$, QI FU ${ }^{3}$, TAO ZHAO ${ }^{2}$, RUI-YING YAN ${ }^{2}$ and XING SU ${ }^{2}$ \\ ${ }^{1}$ Department of Internal Medicine, Weinan Hospital of Traditional Chinese Medicine; ${ }^{2}$ Department of Intensive Care Unit, \\ Weinan Central Hospital Affiliated to Shaanxi University of Chinese Medicine; ${ }^{3}$ Department of Pharmacy, \\ Weinan Central Hospital Affiliated to Shaanxi University of Chinese Medicine, Weinan, Shaanxi 714000, P.R. China
}

Received February 21, 2019; Accepted September 10, 2019

DOI: $10.3892 / \mathrm{etm} .2019 .8153$

\begin{abstract}
Thrombolytic treatment is recommended for patients with high-risk pulmonary embolism. The present study compared thrombolytic therapy with urokinase and reteplase. A total of 37 patients presenting with acute high-risk pulmonary embolism at the Intensive Care Unit of Weinan Central Hospital of Shaanxi Province (Weinan, China) between June 2013 and January 2017 were retrospectively analyzed. According to their treatment, these subjects were assigned to the reteplase group $(n=16)$ or the urokinase group $(n=21)$. Systolic blood pressure (SBP), heart rate (HR) and respiratory rate (RR) were recorded prior to, and at 2, 4, 24 and $48 \mathrm{~h}$ after thrombolytic therapy. Complications, including bleeding, were closely monitored. Changes in blood gas analysis, troponin-T (TNT), pro-B-type natriuretic peptide (pro-BNP) and D-dimer (D-D) were observed. In the reteplase and urokinase group, 11 and 13 cases exhibited marked improvement, treatment was rated as effective in 4 and 6 cases, and 1 and 2 mortalities occurred, resulting in an overall effective rate of 93.8 and $90.5 \%$, respectively $(\mathrm{P}>0.05)$. In the reteplase group, one patient was unsuccessfully resuscitated and died. In the urokinase group, one patient died of gastric hemorrhage after $22 \mathrm{~h}$ of thrombolysis, while another patient died of brain failure resuscitation. The treatment improved the SBP in each of the two groups $(\mathrm{P}<0.05)$, and this outcome was similar between these two groups $(\mathrm{P}>0.05)$. HR and RR were similar prior to and after thrombolytic therapy $(P>0.05)$. In contrast to the urokinase group, TNT was significantly decreased after thrombolyis compared with the baseline in the reteplase group. Complications in the reteplase group were higher, but in contrast to the urokinase group, no life-threatening bleeding occurred. Although reteplase is as effective as urokinase
\end{abstract}

Correspondence to: Mrs. Lan Ma, Department of Intensive Care Unit, Weinan Central Hospital Affiliated to Shaanxi University of Chinese Medicine, Shengli Street, Weinan, Shaanxi 714000, P.R. China

E-mail:1mdr_1314@163.com

Key words: high-risk pulmonary embolism, urokinase, reteplase, thrombolytic treatment, troponin $\mathrm{T}$ in treating high-risk pulmonary embolism, reteplase may reduce myocardial damage.

\section{Introduction}

Pulmonary thromboembolism (PTE) is characterized by pulmonary circulation dysfunction and respiratory dysfunction, which is caused by a thrombus from the venous system or right heart, blocking the pulmonary artery or its branches. Due to its high morbidity and mortality, it has become the third most common cause of death among patients with cardiovascular disease (1).

Anti-coagulant treatment, including the use of low-molecular-weight heparin or fondaparinux, has been suggested for patients with a high or intermediate clinical probability of acute PTE, unless contraindicated, e.g. by severe renal impairment, high bleeding risk, arterial hypotension or extremes in body weight and age (2). However, in cases of high-risk pulmonary embolism, based on the evaluation of the risk of early pulmonary embolism-associated death, treatments to break down or remove the thrombus should be considered (3). For hemodynamically unstable patients with confirmed high-risk PTE, the guidelines suggest immediate thrombolysis treatment (2). Surgical embolectomy or catheter-based thrombus removal are becoming more common. However, even with improved safety and the development of better techniques, its application remains restricted to patients with thrombolysis treatment that is absolutely contraindicated or has failed (3).

For patients with acute high-risk pulmonary embolism, thrombolytic therapy may, relieve symptoms and reduce the requirement for mechanical ventilation. Hence, thrombolytic therapy improves patient outcomes by reducing right-ventricular injury, improving exercise tolerance and preventing the recurrence of pulmonary embolism, thereby improving the survival rate (4). The validated regimens for thrombolytic therapy include urokinase, streptokinase and recombinant tissue plasminogen activator (alteplase) (5). However, at present, there is no preferred thrombolytic method, since these different regimens have similar outcomes (5). Reteplase is a third-generation thrombolytic drug that has yet to be fully validated for treating PTE. Reteplase is also a derivative of tissue-type plasminogen activator, which has been structurally altered to retain a strong fibrin-selective thrombolytic effect (6). This is useful, since fibrin 
is one of the major components of a thrombus (7). Furthermore, reteplase has a reduced binding force with the hepatic scavenger receptor and a prolonged plasma half-life (6). In addition, reteplase may be directly administered by intravenous injection, making its use more convenient (8). On the basis of the significantly improved blood clot penetration and enhanced thrombolytic ability of reteplase in vivo (6), various studies on acute myocardial infarction have revealed that reteplase has a higher vascular recanalization rate when compared to alteplase and streptokinase (9-12).

However, evidence to justify its use in the treatment of pulmonary embolism is currently limited and it has yet to be validated for this type of treatment (13). The aim of the present study was to compare the effectiveness of reteplase with urokinase in treating high-risk pulmonary embolisms. Hence, the outcomes of patients with high-risk PTE were treated with reteplase or urokinase were retrospectively compared.

\section{Materials and methods}

Patients and data. All patients who were rescued by the Intensive Care Unit (ICU) of Weinan Central Hospital of Shaanxi Province (Weinan, China) between June 2013 and January 2017 were retrospectively analyzed. A total of 37 patients with acute high-risk pulmonary embolism were selected for analysis in the present study. Among these patients, 17 were male and 20 were female. The age of the reteplase group was 65.50 (age, 60.25-70.50), and the age of the urokinase group was 65.50 (60.50-70.25). All patients complied with the diagnostic criteria for high-risk pulmonary embolism of either the American Heart Association (14) or the Chinese Society of Cardiology of the Chinese Medical Association on the diagnosis and management of acute pulmonary embolism (15).

All patient data were retrieved from the hospital's database. These data included information from the patient's medical charts, including age, sex, medical history, treatment outcome and complications. The criteria for inclusion in the present study were as follows: i) Adult patients diagnosed with high-risk PTE and ii) patients who underwent intravenous reteplase or urokinase thrombolytic therapy. Patients were excluded if they had any of the following: i) $>2$ weeks after disease onset; ii) incomplete data set; iii) contraindications for thrombolytic treatment (14); iv) other forms of thrombolytic therapy, e.g. catheter-directed thrombolysis; v) severe cardiovascular disease and cerebrovascular disease. The present study conformed to the Declaration of Helsinki and was approved by the Clinical Ethics Committee of Weinan Central Hospital (Weinan, China). Patient consent was obtained at the time of hospitalization.

Therapeutic methods. According to the treatment with different thrombolytic drugs, the subjects were divided into two groups: The reteplase group and the urokinase group. For each group of patients, the respective thrombolytic agent was applied according to the following standard procedures $(5,16)$ : A dose of $18 \mathrm{mg}$ of reteplase (Shandong Ahua Biological Pharmaceutical Co. Ltd.) was diluted in $20 \mathrm{ml}$ saline and intravenously administered to patients in the reteplase group for $10 \mathrm{~min}$, while another dose of $18 \mathrm{mg}$ reteplase was administered to patients after $30 \mathrm{~min}$, resulting in a total dose of $36 \mathrm{mg}$. Urokinase for injection (Tianjin Biochem Pharmaceutical Co. Ltd.) was used at a dose of $20,000 \mathrm{U} / \mathrm{kg}$ for patients in the urokinase group. This was diluted in $40 \mathrm{ml}$ saline and intravenously pumped over $2 \mathrm{~h}$.

After the thrombolytic therapy, the clotting ability was examined every $4 \mathrm{~h}$. For each of the two groups, sequential anti-coagulant therapy with low-molecular-weight heparin calcium (dosage, $0.1 \mathrm{ml} / 10 \mathrm{~kg}$ ) was started when the activated partial thromboplastin time dropped to twice the normal value. In addition to the thrombolytic anti-coagulant therapy, all patients were treated with oxygen therapy. Oxygen therapy or mechanical ventilation was performed according to the hypoxia state: Those patients with a partial pressure of oxygen $\left(\mathrm{PO}_{2}\right)<60 \mathrm{mmHg}$, which improved to $\mathrm{PO}_{2} \geq 60 \mathrm{mmHg}$ after $30 \mathrm{~min}$ of oxygen inhalation, were allowed to continue to inhale oxygen; patients with $\mathrm{PO}_{2}<60 \mathrm{mmHg}$, which remained at $\mathrm{PO}_{2}<60 \mathrm{mmHg}$ after $30 \mathrm{~min}$, were subjected to mechanical ventilation to achieve sufficient oxygen inhalation. The process is presented in Fig. 1. Elevating blood pressure therapy and symptomatic treatment were provided as required.

Routine blood test, blood clotting series, assessment of liver and kidney function, D-dimer (D-D), troponin T (TNT) and pro-B-type natriuretic peptide (pro-BNP), as well as blood gas analysis were performed prior to thrombolysis. The blood gas analysis was performed with arterial blood. The other examinations were performed using venous blood for analysis. D-D was assessed by latex-enhanced immunoturbidimetry (Beijing Strong Biotechnologies Inc.), TNT and pro-BNP were assessed by electrochemiluminescence (F Hoffmann-La Roche Ltd.) and blood gas analysis was performed using an electrode.

Outcome variables. Arterial systolic blood pressure (SBP), heart rate $(\mathrm{HR})$ and change in respiratory rate $(\mathrm{RR})$ were recorded prior to thrombolysis, and at 2, 4, 24 and $48 \mathrm{~h}$ after thrombolytic therapy. The occurrence of complications, including bleeding, was closely monitored. Furthermore, blood gas, TNT, pro-BNP and D-D were measured prior to thrombolysis and at $48 \mathrm{~h}$ after thrombolysis.

According to the criteria for efficacy determination in the Chinese Expert Consensus on the Diagnosis and Management of Acute Pulmonary Embolism 2010 (17), the improvement of dyspnea was evaluated using six levels: i) Cure: The dyspnea and other symptoms completely disappeared according to CT pulmonary angiography, pulmonary perfusion/ventilation scan or catheter-directed pulmonary angiography; ii) marked improvement: The dyspnea and other symptoms were obviously relieved or patients were successfully resuscitated (segments with defective perfusion decreased by 7-9 or the area of the lung with defective perfusion was reduced by $>75 \%$ ); iii) partial improvement: The dyspnea and other symptoms were reduced (the number of segments with defective perfusion decreased by 1-6 or the area of the lung with defective perfusion was reduced by $50-75 \%$ ); iv) no improvement: The dyspnea and other symptoms exhibited no significant changes; v) deterioration: The dyspnea and other symptoms were aggravated; vi) death. The outcomes were independently evaluated by three chief physicians and one deputy chief physician with more than 10 years of experience, and discrepancies were resolved by consensus. Effective thrombolysis was defined as the sum of the cure, marked improvement and partial improvement rates. 
Table I. Baseline characteristics of the patients.

\begin{tabular}{|c|c|c|c|}
\hline Parameter & Reteplase $(n=16)$ & Urokinase $(\mathrm{n}=21)$ & P-value \\
\hline Age (years) & $66.8 \pm 5.5$ & $64.5 \pm 12.2$ & 0.268 \\
\hline Sex (male/female) & $6 / 10$ & $12 / 9$ & 0.236 \\
\hline $\operatorname{BMI}\left(\mathrm{kg} / \mathrm{m}^{2}\right)$ & $24.5 \pm 1.9$ & $24.3 \pm 1.9$ & 0.391 \\
\hline Previous surgery prior to the event & $10(62.5)$ & $13(61.9)$ & 0.970 \\
\hline Orthopedic surgery & $5(31.2)$ & $7(33.3)$ & \\
\hline Cerebral surgery & 0 & $3(14.3)$ & \\
\hline Chest surgery & $2(12.5)$ & $2(9.52)$ & \\
\hline Hepatobiliary surgery & $2(12.5)$ & 0 & \\
\hline Breast surgery & $1(6.25)$ & 0 & \\
\hline Thyroid surgery & 0 & $1(4.76)$ & \\
\hline $\mathrm{SBP}(\mathrm{mmHg})$ & $77.0 \pm 49.7$ & $89.3 \pm 37.2$ & 0.264 \\
\hline HR (bpm) & $80.2 \pm 60.2$ & $75.0 \pm 42.4$ & 0.410 \\
\hline RR (bpm) & $21.7 \pm 15.3$ & $20.1 \pm 9.9$ & 0.389 \\
\hline $\mathrm{PO}_{2} / \mathrm{FiO}_{2}(\mathrm{mmHg})$ & $178.3 \pm 62.0$ & $167.3 \pm 97.0$ & 0.388 \\
\hline TNT (ng/l) & $0.11 \pm 0.12$ & $0.23 \pm 0.28$ & 0.124 \\
\hline D-D (ng/l) & $13,931 \pm 24,282$ & $28,129 \pm 32,519$ & 0.161 \\
\hline Pro-BNP (pg/ml) & $2,488 \pm 2,571$ & $3,617 \pm 4,388$ & 0.280 \\
\hline Uric acid $(\mu \mathrm{mol} / \mathrm{l})$ & $299 \pm 100$ & $352 \pm 106$ & 0.085 \\
\hline Blood glucose (mmol/l) & $6.40 \pm 1.70$ & $5.91 \pm 1.23$ & 0.185 \\
\hline
\end{tabular}

Values are expressed as the mean \pm standard deviation or $\mathrm{n}(\%)$. BMI, body mass index; SBP, systolic blood pressure; HR, heart rate; RR, respiratory rate; $\mathrm{PO}_{2} / \mathrm{FiO}_{2}$, oxygenation index; TNT, troponin T; D-D, D-dimer; Pro-BNP, pro-B-type natriuretic peptide.

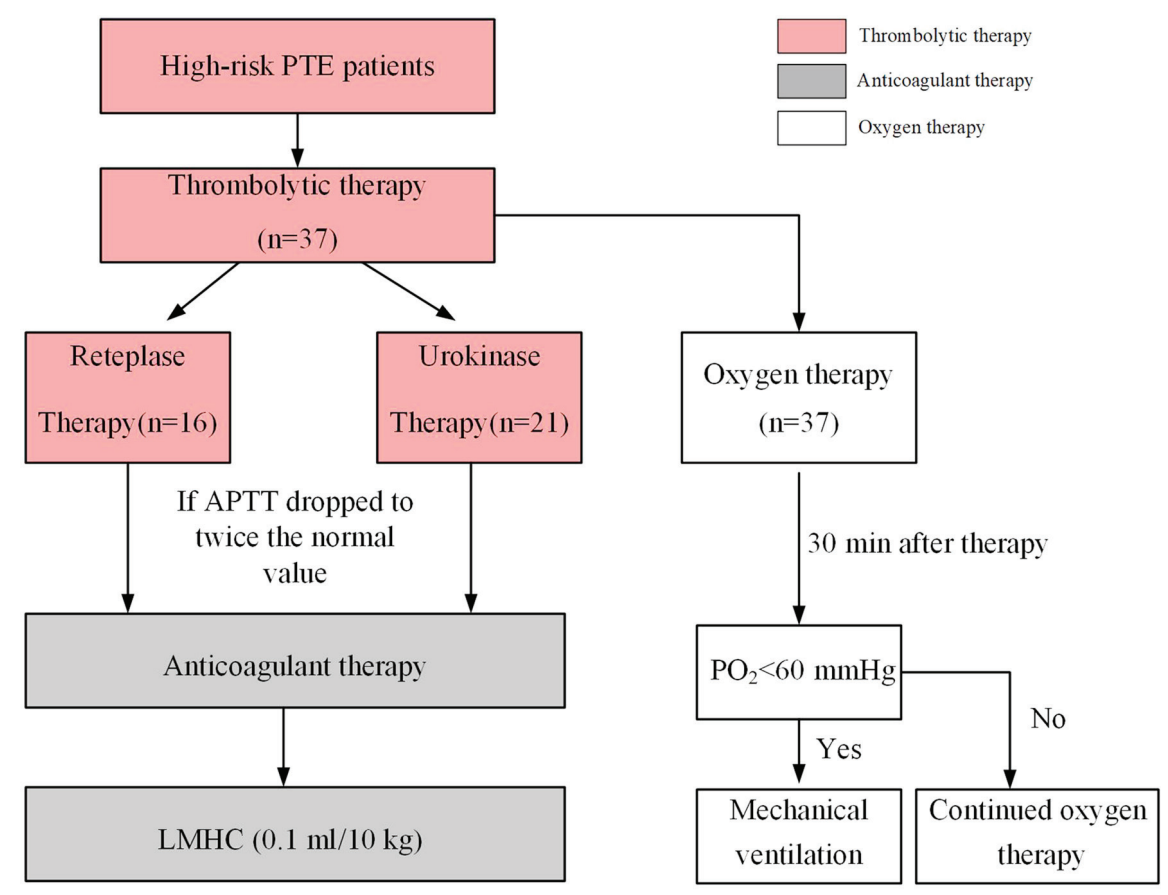

Figure 1. Administration protocol of the present study. PTE, pulmonary thromboembolism; APTT, activated partial thromboplastin time; LMHC, low-molecular-weight heparin calcium; $\mathrm{PO}_{2}$, partial oxygen pressure.

Statistical analysis. Statistical analysis was performed using SPSS 19.0 software (IBM Corp.). The normality of distribution was evaluated using the Kolmogorov-Smirnov test. Variables with a normal distribution were compared using Student's t-test and their values are expressed as the mean \pm standard deviation. Categorical variables were presented as the frequency (percentage). The data in Table I were analyzed between groups using the Chi-square and the 
Table II. Comparison of thrombolytic effects between the reteplase and urokinase groups.

\begin{tabular}{lcc}
\hline Item & Reteplase $(\mathrm{n}=16)$ & Urokinase $(\mathrm{n}=21)$ \\
\hline Thrombolytic effect & & 0 \\
Cure & 0 & $13(61.9)$ \\
Marked improvement & $11(68.8)$ & $6(28.6)$ \\
Partial improvement & $4(25.0)$ & 0 \\
No improvement & 0 & 0 \\
Deterioration & 0 & $2(9.5)$ \\
Death & $1(6.2)$ & $1(4.8)$ \\
Major bleeding complications & 0 & $2(9.5)$ \\
Minor bleeding complications & $6(37.5)$ & 90.5
\end{tabular}

Values are expressed as n (\%) unless otherwise specified. The data were compared between groups using the Chi-Square test.

Table III. Comparison of $\mathrm{PO}_{2} / \mathrm{FiO}_{2}$, TNT, D-D and pro-BNP prior to and after treatment (48 h) in the two groups.

\begin{tabular}{|c|c|c|c|}
\hline Variable & Reteplase $(n=16)$ & Urokinase $(\mathrm{n}=2)$ & P-value \\
\hline \multicolumn{4}{|c|}{$\mathrm{PO}_{2} / \mathrm{FiO}_{2}(\mathrm{mmHg})$} \\
\hline Baseline & $178.3 \pm 62.0$ & $167.3 \pm 97.0$ & 0.914 \\
\hline $48 \mathrm{~h}$ & $242.6 \pm 104.9$ & $269.8 \pm 80.7^{\mathrm{a}}$ & 0.583 \\
\hline \multicolumn{4}{|c|}{ Pro-BNP (pg/ml) } \\
\hline Baseline & $2,488 \pm 2,571$ & $3,617 \pm 4,388$ & 0.473 \\
\hline $48 \mathrm{~h}$ & $967 \pm 1,067$ & $2,673 \pm 2,877$ & 0.190 \\
\hline \multicolumn{4}{|l|}{ TNT $(\mu \mathrm{g} / \mathrm{l})$} \\
\hline Baseline & $0.111 \pm 0.116$ & $0.226 \pm 0.277$ & 0.963 \\
\hline $48 \mathrm{~h}$ & $0.016 \pm 0.009^{\mathrm{a}}$ & $0.991 \pm 2.610$ & 0.078 \\
\hline \multicolumn{4}{|l|}{$\mathrm{D}-\mathrm{D}(\mu \mathrm{g} / \mathrm{l})$} \\
\hline Baseline & $13,931 \pm 24,282$ & $28,129 \pm 32,519$ & 0.080 \\
\hline $48 \mathrm{~h}$ & $2,342 \pm 1,224$ & $5,313 \pm 3,547^{a}$ & 0.889 \\
\hline
\end{tabular}

Values are expressed as the mean \pm standard deviation. The data were compared by two-way analysis of variance, followed by Sidak's multiple-comparisons test. ${ }^{a} \mathrm{P}<0.05$, vs. baseline. $\mathrm{PO}_{2} / \mathrm{FiO}_{2}$, oxygenation index; TNT, troponin T; D-D, D-dimer; Pro-BNP, pro-B-type natriuretic peptide.

t-test. The data in Table II were analyzed between groups using the Chi-square test. The data in Table III were compared by two-way analysis of variance (ANOVA), followed by Sidak's multiple-comparisons test. Two-sided P-values $<0.05$ were considered to indicate statistical significance. The data in Fig. 2 were analyzed using repeated-measures ANOVA with the time being the within factor and treatment (reteplase and urokinase) as the between factor. Bonferroni-adjusted post-hoc comparison was used to identify differences between paired data.

\section{Results}

Baseline characteristics. A total of 37 patients were available for analysis in the present study and the age of these patients ranged from 28 to 86 years (average age, $65.5 \pm 11.5$ years).
Among those patients, 16 were treated with reteplase, while 21 patients were treated with urokinase. Trauma and bed rest after surgery were the major causes of pulmonary embolism, accounting for $62.2 \%$ of cases. The other causes were hypertension $(8.1 \%)$, diabetes $(8.1 \%)$, cerebral infarction $(5.4 \%)$, coronary heart disease $(5.4 \%)$ and lower extremity varicose veins (10.8\%; data not shown). However, there was no significant difference in the baseline characteristics between the two groups (Table I).

Patient outcomes. As presented in Table II, the proportion of patients with a marked and partial improvement was comparable between the two groups. The overall effective thrombolysis rate was $93.8 \%(15 / 16)$ and $90.5 \%$ (19/21) for the reteplase and urokinase group, respectively, but the difference was not statistically significant $(\mathrm{P}>0.999)$. 


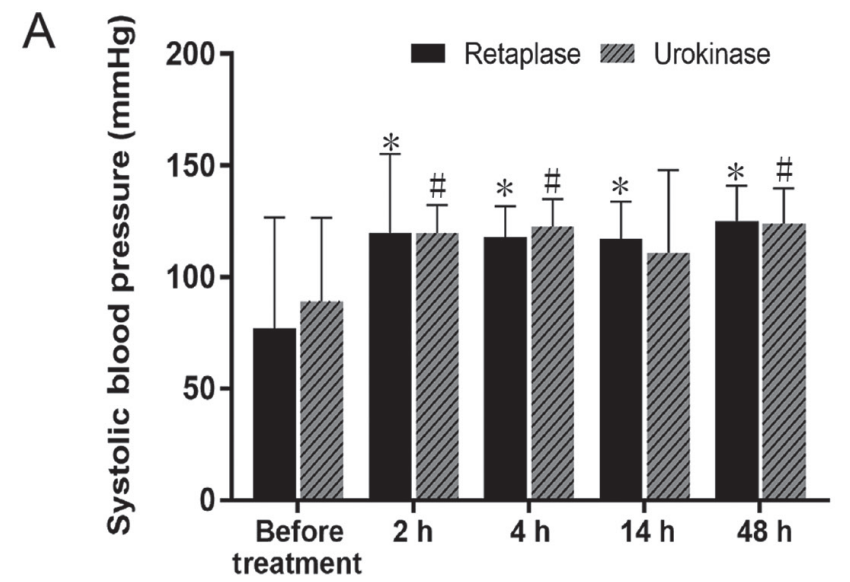

B
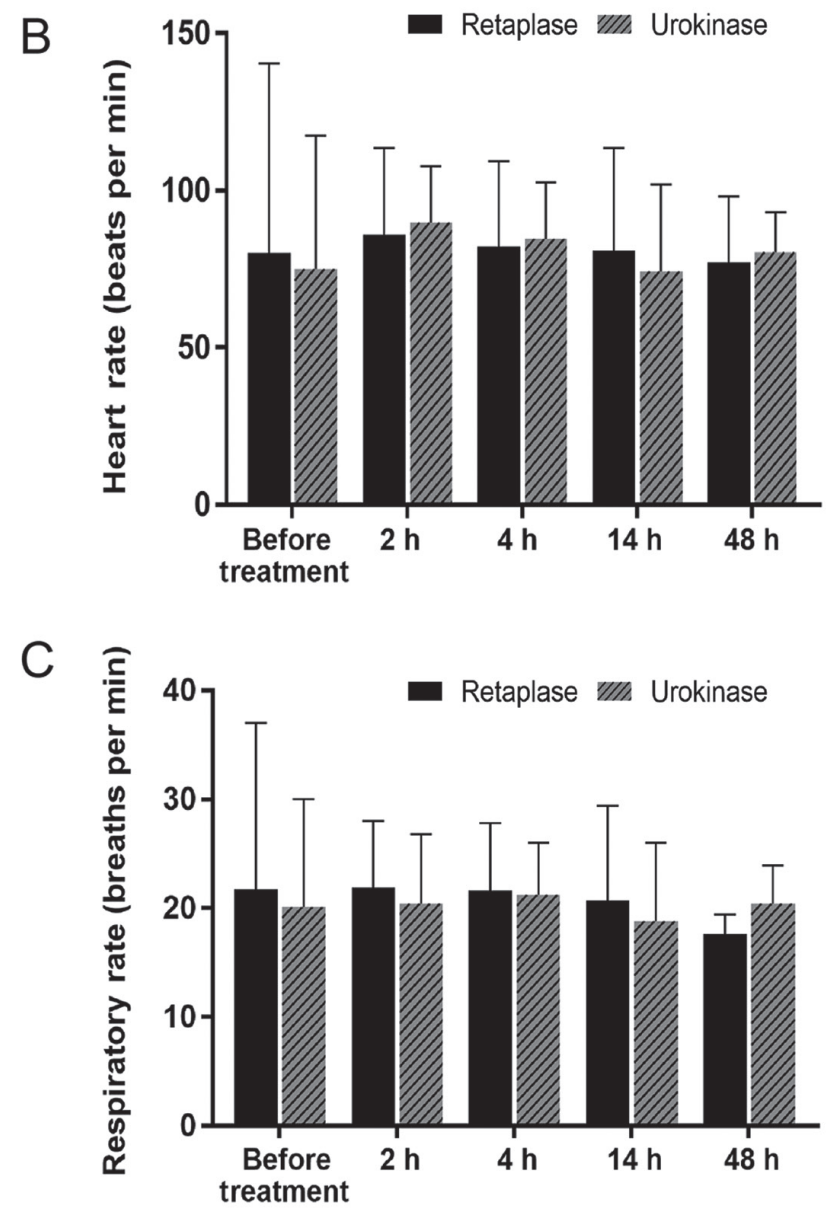

Figure 2. Changes in vital variables after reteplase and urokinase thrombolytic therapy in patients with high-risk pulmonary embolism. (A) Changes in systolic blood pressure; (B) Changes in heart rate; (C) Changes in respiratory rate. Values are expressed as the mean \pm standard deviation. ${ }^{*} \mathrm{P}<0.05$ vs. prior to treatment for the reteplase group; ${ }^{\#} \mathrm{P}<0.05$ vs. prior to treatment for the urokinase group.

The treatment significantly improved the SBP in each of the two groups, but there was no significant difference between these two groups prior to and after thrombolytic therapy (Fig. 2A). Furthermore, the difference in HR and RR of patients in each of these two groups was not statistically significant prior to and after thrombolytic therapy, and there was no significant difference between these two groups (Fig. 2B and C).
There was no significant difference in $\mathrm{PO}_{2} /$ fraction of inspired oxygen $\left(\mathrm{FiO}_{2}\right)$, pro-BNP and $\mathrm{D}-\mathrm{D}$ prior to and after treatment in the reteplase group, but TNT significantly decreased after treatment $(\mathrm{P}=0.046)$. There was a significant difference in $\mathrm{PO}_{2} / \mathrm{FiO}_{2}$ and $\mathrm{D}$-D prior to vs. after thrombolysis in the urokinase group $(\mathrm{P}=0.007$ and 0.001 , respectively), but there was no significant difference in TNT and pro-BNP. Furthermore, there was no significant difference in $\mathrm{PO}_{2} / \mathrm{FiO}_{2}$, pro-BNP, TNT and D-D between the two groups after thrombolysis. TNT exhibited a downward trend after thrombolysis in the reteplase group, while TNT exhibited an upward trend after thrombolysis in the urokinase group (Table III). The difference in the other indicators was not statistically significant.

Complications during treatment. The hospital stay in the reteplase group was $7.00(5.25-8.75)$ days, and the hospital stay in the urokinase group was 8.00 (6.25-10.00) days. In the reteplase group, three patients had subcutaneous ecchymosis at the puncture site, which was absorbed without any special treatment. Furthermore, one patient had epistaxis and errhysis at the puncture site, one patient had increased bleeding from their drainage tube on the second day after the operation and one patient had pseudoaneurysm due to a short compression time after elbow artery puncture, which was performed prior to thrombolytic therapy. These three complications improved after enhanced hemostasis by compression. Furthermore, one patient in the reteplase group had an unsuccessful resuscitation and died. In the urokinase group, all initial resuscitations were successful. One patient had epistaxis, while one patient had errhysis at the puncture site, and these two patients improved after hemostasis by compression. Furthermore, one patient died of gastric hemorrhage after $22 \mathrm{~h}$ of thrombolysis and one patient died of brain failure after 11 days of resuscitation. Overall, the occurrence of complications in the reteplase group was higher when compared with that in the urokinase group, but no life-threatening bleeding occurred. However, life-threatening bleeding occurred in the urokinase group.

\section{Discussion}

Pulmonary embolism occurs when a detached thrombus (embolism) from any part of the venous territory becomes lodged in the pulmonary artery. Although the origin of the embolism may be venous thrombosis in any location (upper extremities, prostatic, uterine and renal veins and right heart chambers), it is a lower extremity deep vein thrombosis in most cases (90-95\%), which is frequently asymptomatic. High-risk pulmonary embolism, previously referred to as massive pulmonary embolism, is characterized by hypotension or shock, accounts for $\sim 5 \%$ of cases and is associated with early mortality in at least $15 \%$ of cases. Thrombolytic treatment is usually recommended for these patients (18).

In recent years, due to the in-depth understanding and improvement in diagnostic methods for PTE, the number of diagnoses have rapidly increased. For patients with high-risk pulmonary embolism, thrombolytic therapy may restore lung perfusion early, relieve symptoms, reduce the requirement for mechanical ventilation, reduce right-ventricular injury and improve exercise tolerance, which effectively prevents the 
recurrence of pulmonary embolism and improves the survival rate $(1,2,15,18)$. In the present study, reteplase and urokinase were used for the treatment of high-risk pulmonary embolism, and each of the two drugs achieved good effects by rapidly improving the patient's subjective symptoms and stabilizing their hemodynamics. Although the results were similar for the two groups of patients, it was suggested that the use of reteplase may reduce myocardial damage and prevent myocardial damage when compared with urokinase, with fewer major bleeding episodes. Therefore, the present study provides clinical experience for the further application of reteplase in patients with acute high-risk pulmonary embolism and suggests that it may be appropriate to broaden the application range of reteplase.

The results of the present study indicated that in terms of effectiveness, there was no significant difference between reteplase and urokinase. The two groups exhibited high levels of effectiveness and these results were supported by previous evidence suggesting that thrombolytic therapy is beneficial for high-risk PTE patients $(4,5)$ and guidelines that support the use of thrombolysis in high-risk patients $(18,19)$.

To date, certain studies have revealed significant differences in outcome among different thrombolytic agents (5,20-24). In the present study, biomarkers and clinical parameters were compared in order to determine whether a difference exists between the reteplase and urokinase treatment groups. The results revealed only few significant differences between these two groups in certain biomarkers and clinical parameters. The improvement of TNT after thrombolysis was significant in the reteplase group, while the improvement of $\mathrm{PO}_{2} / \mathrm{FiO}_{2}$ and D-D was significant in the urokinase group, but the other indicators were not significantly different between the two drug treatment groups. TNT is a common marker of myocardial damage (25), $\mathrm{PO}_{2} / \mathrm{FiO}_{2}$ indicates the level of respiratory distress and D-D is a product of fibrolysis (26). In general, these similar results may be due to the small number of patients enrolled in the present study, preventing the differences from reaching a significant level. However, it is noteworthy that TNT continued to exhibit an upward trend after the thrombolytic therapy with urokinase, while TNT declined in the reteplase group, suggesting that reteplase may be better for improving myocardial damage.

The major concern with thrombolysis is the occurrence of complications, the most common of which is major bleeding (5). In the present study, complications of bleeding increased after treatment in the reteplase group, but no fatal bleeding occurred. However, one patient died of respiratory cardiopulmonary arrest due to the pulmonary embolism itself, which was not associated with reteplase. In the urokinase group, complications of fatal bleeding increased after the treatment. Furthermore, one patient treated with urokinase developed gastrointestinal hemorrhage and died, while another patient died of the pulmonary embolism itself (death from brain failure after respiratory arrest). In the present study, the complications increased following application of reteplase, which is similar to previous results (14), but different from the results reported by Liu et al (27). The possible reason may be that the present study is a single-center retrospective study and the sample size was small.

The present study has certain limitations. First, the small sample size resulted from the single-center nature of the study. Data from multiple centers may increase the power of evidence of the results. In order to reach exact conclusions, the sample size requires to be increased in future studies. In addition, the present study was retrospective in nature. Hence, the patients were not allocated to groups in a randomized manner. Therefore, this may have introduced a certain level of bias. Furthermore, due to lack of bedside echocardiography equipment at the ICU department in the early years, no echocardiographic data were available for all patients. In future prospective studies by our group, inclusion of these parameters will be considered. Furthermore, the patients were not followed up after discharge. Hence, it was not possible to establish the long-term outcomes for these patients.

In conclusion, reteplase, a third-generation thrombolytic drug, has not been previously validated for the treatment of pulmonary embolism. The results of the present study suggest that, although reteplase is similar to urokinase in terms of thrombolytic effectiveness for patients with high-risk pulmonary embolism, it may also reduce myocardial damage.

\section{Acknowledgements}

Not applicable.

\section{Funding}

No funding was received.

\section{Availability of data and materials}

The datasets used and/or analyzed during the current study are available from the corresponding author on reasonable request.

\section{Authors' contributions}

LM conceived and designed the study and prepared the manuscript. YZ, QF and TZ made substantial contributions to acquisition and interpretation of data. R-YY and XS analyzed the data. All authors read and approved the manuscript.

\section{Ethics approval and consent to participate}

The present study was approved by the Clinical Ethics Committee of Weinan Central Hospital (Weinan, China).

\section{Patient consent for publication}

Not applicable.

\section{Competing interests}

The authors declare that they have no competing interests.

\section{References}

1. Meyer G: Effective diagnosis and treatment of pulmonary embolism: Improving patient outcomes. Arch Cardiovasc Dis 107: 406-414, 2014.

2. Chalikias $\mathrm{G}$ and Konstantinides S: Acute phase treatment of pulmonary embolism. Curr Vasc Pharmacol 12: 393-400, 2014. 
3. Streiff MB, Agnelli G, Connors JM, Crowther M, Eichinger S, Lopes R, McBane RD, Moll S and Ansell J: Guidance for the treatment of deep vein thrombosis and pulmonary embolism. J Thromb Thrombolysis 41: 32-67, 2016.

4. Marti C, John G, Konstantinides S, Combescure C, Sanchez O, Lankeit M, Meyer G and Perrier A: Systemic thrombolytic therapy for acute pulmonary embolism: A systematic review and meta-analysis. Eur Heart J 36: 605-614, 2015.

5. Stone J, Hangge P, Albadawi H, Wallace A, Shamoun F, Knuttien MG, Naidu S and Oklu R: Deep vein thrombosis: Pathogenesis, diagnosis, and medical management. Cardiovasc Diagn Ther 7 (Suppl 3): S276-S284, 2017.

6. Marcos-Contreras OA, Ganguly K, Yamamoto A Shlansky-Goldberg R, Cines DB, Muzykantov VR and Murciano JC: Clot penetration and retention by plasminogen activators promote fibrinolysis. Biochem Pharmacol 85: 216-222, 2013

7. Sadowski M, Ząbczyk M and Undas A: Coronary thrombus composition: Links with inflammation, platelet and endothelial markers. Atherosclerosis 237: 555-561, 2014.

8. Smalling RW, Bode C, Kalbfleisch J, Sen S, Limbourg P, Forycki F, Habib G, Feldman R, Hohnloser S and Seals A: More rapid, complete, and stable coronary thrombolysis with bolus administration of reteplase compared with alteplase infusion in acute myocardial infarction. RAPID Investigators. Circulation 91: 2725-2732, 1995.

9. Rosenberg DG, Levin E, Lausell A, Brown A, Gardner J, Perez E, Veenendaal M, Ong YS and Gunn M: Feasibility and timing of prehospital administration of reteplase in patients with acute myocardial infarction. J Thromb Thrombolysis 13: 147-153, 2002.

10. Morrow DA, Antman EM, Sayah A, Schuhwerk KC Giugliano RP, deLemos JA, Waller M, Cohen SA, Rosenberg DG, Cutler SS, et al: Evaluation of the time saved by prehospital initiation of reteplase for ST-elevation myocardial infarction: Results of the early retavase-thrombolysis in myocardial infarction (ER-TIMI) 19 trial. J Am Coll Cardiol 40: 71-77, 2002.

11. Luiz T, Wilhelms A, Madler C, Pollach G, Haaff B, Grüttner J and Viergutz T: Outcome of out-of-hospital cardiac arrest after fibrinolysis with reteplase in comparison to the return of spontaneous circulation after cardiac arrest score in a geographic region without emergency coronary intervention. Exp Ther Med 13: 1598-1603, 2017

12. Adivitiya and Khasa YP: The evolution of recombinant thrombolytics: Current status and future directions. Bioengineered 8: 331-358, 2017.

13. Lippi G, Mattiuzzi C and Favaloro EJ: Novel and emerging therapies: Thrombus-targeted fibrinolysis. Semin Thromb Hemost 39: 48-58, 2013.

14. Jaff MR, McMurtry MS, Archer SL, Cushman M, Goldenberg N, Goldhaber SZ, Jenkins JS, Kline JA, Michaels AD, Thistlethwaite $\mathrm{P}$, et al: Management of massive and submassive pulmonary embolism, iliofemoral deep vein thrombosis, and chronic thromboembolic pulmonary hypertension: A scientific statement from the American Heart Association. Circulation 123: 1788-1830, 2011.

15. Pulmonary Circulation and Right Ventricular Function Assembly of Chinese Society of Cardiology of Chinese Medical Association: Chinese expert consensus on the diagnosis and management of acute pulmonary embolism (2015). Zhonghua Xin Xue Guan Bing Za Zhi 44: 197-211, 2016 (In Chinese).
16. Simpson D, Siddiqui MA, Scott LJ and Hilleman DE: Reteplase: A review of its use in the management of thrombotic occlusive disorders. Am J Cardiovasc Drugs 6: 265-285, 2006.

17. Department of Pulmonary Vascular Diseases, Chinese Medical Association Cardiovascular Disease: Chinese expert consensus on the diagnosis and management of acute pulmonary embolism. Chin J Intern Med 49: 74-81, 2010 (In Chinese).

18. Konstantinides SV, Torbicki A, Agnelli G, Danchin N, Fitzmaurice D, Galiè N, Gibbs J, Huisman MV, Humbert M, Kucher N, et al: Authors/task force members. corrigendum to: 2014 ESC Guidelines on the diagnosis and management of acute pulmonary embolism. Eur Heart J 36: 2642, 2015.

19. Torbicki A, Perrier A, Konstantinides S, Agnelli G, Galiè N, Pruszczyk P, Bengel F, Brady AJ, Ferreira D, Janssens U, et al: ESC committee for practice guidelines (CPG). Guidelines on the diagnosis and management of acute pulmonary embolism: The task force for the diagnosis and management of acute pulmonary embolism of the European society of cardiology (ESC). Eur Heart J 29: 2276-2315, 2008

20. Bloomer TL, El-Hayek GE, McDaniel MC, Sandvall BC, Liberman HA, Devireddy CM, Kumar G, Fong PP and Jaber WA: Safety of catheter-directed thrombolysis for massive and submassive pulmonary embolism: Results of a multicenter registry and meta-analysis. Catheter Cardiovasc Interv 89: 754-760, 2017.

21. Daley MJ, Murthy MS and Peterson EJ: Bleeding risk with systemic thrombolytic therapy for pulmonary embolism: Scope of the problem. Ther Adv Drug Saf 6: 57-66, 2015.

22. Long B and Koyfman A: Current controversies in thrombolytic use in acute pulmonary embolism. J Emerg Med 51: 37-44, 2016.

23. Nagamalesh UM, Prakash VS, Naidu KCK, Sarthak S, Hegde AV and Abhinay T: Acute pulmonary thromboembolism: Epidemiology, predictors, and long-term outcome-A single center experience. Indian Heart J 69: 160-164, 2017.

24. Goldhaber SZ, Kessler CM, Heit J, Markis J, Sharma GV, Dawley D, Nagel JS, Meyerovitz M, Kim D and Vaughan DE: Randomised controlled trial of recombinant tissue plasminogen activator versus urokinase in the treatment of acute pulmonary embolism. Lancet 2: 293-298, 1988.

25. Mannu GS: The non-cardiac use and significance of cardiac troponins. Scott Med J 59: 172-178, 2014.

26. Olson JD: D-dimer: An overview of hemostasis and fibrinolysis, assays, and clinical applications. Adv Clin Chem 69: 1-46, 2015.

27. Liu XY, Zhang Y, Li MW, Wang XP, Qi DD, Hao PY, Zhang H, Cheng QQ, Zhao LS, Gao CY and Hu DY: Efficacy of thrombolytic therapy using reteplase in cases with acute ST-segment elevation myocardial infarction: Results from a multicenter clinical trial. Zhonghua Xin Xue Guan Bing Za Zhi 44: 766-770, 2016 (In Chinese).

This work is licensed under a Creative Commons Attribution-NonCommercial-NoDerivatives 4.0 International (CC BY-NC-ND 4.0) License. 